\title{
Tele-nursing strategies in Iran: A narrative literature review
}

Parisa Fathizadeh Dehkordi ${ }^{1}$, Haydeh Heidari ${ }^{*}, 2$, Reza Masoodi ${ }^{2}$, Morteza Sedehi ${ }^{3}$, Fereidoun Khajeali $^{2}$

${ }^{1}$ Community-Oriented Nursing Midwifery Research Center, Shahrekord University of Medical Sciences, Shahrekord, Iran.

${ }^{2}$ School of Nursing and Midwifery, Shahrekord University of Medical Sciences, Shahrekord, Iran.

${ }^{3}$ Department of Epidemiology and Biostatistics, School of Health, Shahrekord University of Medical Sciences, Shahrekord, Iran.

*. Corresponding author: Haydeh Heidari, School of Nursing and Midwifery, Shahrekord University of Medical Sciences, Shahrekord, Iran, Tel: +983833335652, E-mail: haydehheidari@gmail.com.

Cite this article: Fathizadeh Dehkordi, P.; Heidari, H.; Masoodi, R.; Sedehi, M.; Khajeali, F. Tele-nursing strategies in Iran: A narrative literature review. Int $\mathbf{J}$ Epidemiol Health Sci 2020;1(3): e03. Doi: 10.51757/IJEHS.1.3.2020.46189.

\begin{abstract}
Introduction: Tele-nursing is a modern way of promoting quality of nursing care, treatment outcome, reducing medical costs and the need for visits, patient and family involvement in decision making, and careful patient monitoring by using all kinds of technologies. The aim of this article was to determine the most popular method in Iran and the potential reasons.

Methods: In this narrative literature review, PubMed, Science Direct, Google Scholar, SID, and Nindex were searched for full texts of Iranian studies published in English or Persian by using the descriptors of Tele-nursing, MHealth, nursing care, Tele-care, and follow-up with phone calls between 2010 and 2020. The search found 472 articles and after considering the eligibility of articles, 56 articles were included.

Results: The review found that $74 \%$ of papers used the telephone for telenursing and the second popular method was short message service (SMS) followed by internet and smartphone software.

Conclusion: Telenursing includes many methods that nurses can use for distance nursing care which is not limited to telephone or SMS. In Iran, about 68 million people have a smartphone and have access to the internet. Nurses can use this potential capacity to improve nursing care.
\end{abstract}

Keywords: Telenursing, MHealth, Nursing care, Telecare, follow-up with phone calls

\section{Introduction}

Nurses are at the first line of communication with patients in health care systems through planning, coordinating, providing, and evaluating care (1). Nursing improves the quality of the health care. One of the best methods to reach this goal, is Tele-nursing (2), which has commonly been used since 1980 (3).
Tele-nursing is a subset of tele-medicine (4) and the most important way for reaching modern nursing care, to improve the quality of care and quick access to nursing care services by overcoming geographical barriers (5) which it focuses on patients' long-term wellness and health as well as empowerment of nurses with the opportunity of 
educating, follow-up and family support through using communication technologies such as telephones, short message service (SMS), e-learning tools, e-mail (6) and smartphone software (7).

The telephone is the most popular communication tool used in the developed countries due to its cost-effectiveness (3) and easy accessibility (8). Research indicated that telephone consulting is an alternative to in-person visits for providing support and affect behavior changes (9) which it is an effective medium to facilitate the care of chronic patients as well as increase patients' self-care ability by offering medical support and information (10) and by monitoring calls (11).

SMS is one of the mobile phone services (12) and another way of tele-nursing, this method is an interactive service, simple, fast and confidential way for enabling users to send and receive text messages to and from mobile phones and allows rapid reception and reply at low cost (13). Research on SMS in health care services have been focused on two main areas: behavior change interventions and reminders, which can also lead to behavior changes (14).

The computer is another useful device for tele-nursing that can be used for online or offline nursing care. Multimedia CD-DVD is an offline method that transfers education information by Compact Disk (CD) or Digital Video Disk (DVD). The advantages of this method are individual training and no restrictions on the time and duration of the training (15).

There are many types of online methods that can be used by nurses. For example, e-learning is a teaching method based on the web that includes decentralization of the teaching process and individualized learning, flexible access at any time or place, promotion of active learning and can be done by many electronic devices $(16,17)$.

Education software is another method for tele-nursing which has affected families life after the advent of computers and the growing expansion of communication technology and has the ability to transfer medical information like voice, movie, image, and animation (4). It can facilitate data collection and processing for nurses, extend nursing care standards and help nurses for better decision-making (18).

E-mail, as another mode of tele-nursing, has been used for data collection (19) and interview (20). For instance, Kawaguchi and colleagues (in 2004) have used email and video-email for information collection by filling a visual analog scale from and a USB-connected PC camera record, they concluded that this kind of tele-nursing can improve patient's condition (21). In the pilot study conducted by SouzaJunior, it is found that tele-nursing interventions can achieve important results as a way to improve patients' health condition (22). In addition, in a systematic review done by Ghoulami Shilsari and colleagues (in 2019) tele-nursing has been introduced an effective method in nursing performances and promoting selfcare of patients (2).

Currently, the penetration rate of mobile phone subscribers in Iran has been announced 91.2 with the population of 7.1 million people and considering the above index, it can be concluded that there are now more than 68 million mobile phone subscriptions in Iran (23). According to Tehran Times, the number of broadband internet subscribers until the end of september 2018, was about 61 million people with mobile internet access (24).

Previous studies have categorized mobile usage in three groups: 1- Usage by students and health workers, 2- The risk of mobile usage and its wave impact on humankind and animals, 3- Mobile usages for health improvement interventions (25). Also, studies showed that tele-nursing can effectively improve the outcomes of various diseases such as asthma (26), diabetes (27), insomnia (28), hypertension (29), and some mental disorders such as anxiety (30) and depression (31). Ajalli and Fallahi Khoshknab have evaluated the outcomes of telenursing through a systematic review in 2015 (32) and reported that there was not any study about its strategies. So, the objective of this study was to provide a narrative review of tele-nursing strategies in Iran and to find the most popular methods and its potential reason for the method selection.

\section{Materials and methods}

\section{Search strategy}

An extensive literature search was conducted in May 2020 using the PubMed, Science Direct, Google scholar, SID, Nindex. This review was limited to studies that have been done in Iran between 2010 to 2020 in English or Persian language. Inclusion criteria were studies available in full texts, evaluating the use of tele-nursing as a nursing care. Exclusion criteria were using communication technology in a nonnursing care, letters to the editor, qualitative and, and review articles. Both randomized control trials (RCTs) and non-RCTs were included. The tele-nursing strategies, databases, search items, inclusion and exclusion criteria can be seen in Table 1 . 
Table 1. Search strategy

\begin{tabular}{|c|c|c|c|c|}
\hline $\begin{array}{l}\text { Tele-nursing } \\
\text { strategy }\end{array}$ & Database & Search terms & Inclusion criteria & Exclusion criteria \\
\hline $\begin{array}{l}\text {-Tele-nursing } \\
\text {-MHealth } \\
\text {-Nursing care } \\
\text { - Telecare } \\
\text {-Follow-up with } \\
\text { phone calls }\end{array}$ & $\begin{array}{c}\text { PubMed } \\
\text { Science Direct } \\
\text { Google Scholar } \\
\text { SID } \\
\text { Nindex }\end{array}$ & $\begin{array}{c}\text { All (*tele-nursing } \\
\text { and Iran* OR } \\
\text { *telecare and Iran* } \\
\text { OR *follow up } \\
\text { with phone calls } \\
\text { and Iran* OR } \\
\text { *mHealth and } \\
\text { Iran*) AND } \\
\text { all }(* \text { nursing care*) } \\
\text { In English and } \\
\text { Persian }\end{array}$ & $\begin{array}{l}\text { - Available in full- } \\
\text { texts } \\
\text { - Evaluating the use } \\
\text { of tele-nursing as a } \\
\text { nursing care }\end{array}$ & $\begin{array}{c}\text { - Using } \\
\text { communication } \\
\text { technology, a non- } \\
\text { nursing care } \\
\text { - Letters to editor } \\
\text { - Qualitative and } \\
\text { review ones }\end{array}$ \\
\hline
\end{tabular}

Titles, abstracts, and reference lists of the selected studies were also reviewed to examine other potential relevant studies. First, all the titles and abstracts were reviewed for relevance to the topic and to see if they met inclusion criteria. Second, the studies were read and categorized by methodology, tele-

\section{Characteristics of studies reviewed}

Author, year, sample size, type of intervention, Study design, goal, and outcome were extracted to describe the characteristics of the paper (Table 2). The number of articles has increased over time and the peak of publication on this topic was $2016-2017(35.72 \%)$ and $48.21 \%$ of studies were designed as RCT. Investigation of nearly $53.5 \%$ of articles was implemented in 3 months, $21.4 \%$ in 2

\section{Discussion}

Tele-nursing is considered as informational and communicational technology, which can be provided by variety of technologies like telephone, SMS, video, email and internet. The result of this narrative literature review demonstrated that telenursing is a good and economically attractive method for nursing goals of monitoring, educating, follow-up and empowerment of patients. Furthermore, the most popular strategy for using tele-nursing in Iran was telephone $(74 \%)$, probably because of the ease of use among the elderly who often have difficulty working with internet-requiring devices. Despite having national social messengers such as Soroush, Eitaa and bale, the only social messenger that has been used for tele-nursing in Iran is Telegram, which is not available nursing, used strategy, goal, and outcome of each study (Figure 1). The initial search found 472 articles which after removing duplicated articles and nonrelevant ones, 56 articles met inclusion criteria after full-text review.

months and the investigation time for other articles differed from 3 days to 10 weeks. Method of $66 \%$ of the articles was only follow-up after an educational or empowerment program, $12.5 \%$ follow-up with education or consultation and $21.5 \%$ education, consultation or notification (Table 3). The most common methods of tele-nursing were telephone, SMS and software, respectively (Table 4)

for all because of filtering. There was no study using Email in Iran.

Despite the high capacity for sending educational programs to families and nurses and consulting them (33), Hawkins (in 2018) used email for nursing interviews and concluded that it may increase the dependability and confirmability of findings (20). In another study, Kawaguchi used email as an intervention for improvement of chronic conditions (such as blood pressure and blood glucose) and reported the usefulness of this method (21); however, in Iran, this method has been ignored, maybe because email needs computer access, consistent internet connectivity and the most important reason would be the fact that communication through email is not possible for many elder people in Iran. 


\section{Methods of tele-nursing in Iran}

The follow-up after an educational or empowerment program in Iran, was the most popular method for tele-nursing (66\%) (Table 3). It provides training, monitoring, data collection, monitoring care and protection of patients against side effects of medications and chronic conditions with the followup, as a necessary part of nursing care of patients and their family without time and place restrictions (34).

\section{Limitations}

Studies were only selected from full-text articles that were accessible to inspect in Iran which may have restricted the results.

\section{Conclusion}

Tele-nursing is one of the best ways for helping patients even in rural areas and improving the level of nursing care services, but it is not limited to telephone; so, for better utilization of tele-nursing, it is recommended to use another kind of technologies as well. It is suggested to make the best utilization of the wide range of mobile and internet access among Iranians by native social messengers and smartphone software.

\section{Acknowledgement}

This article is a part of the thesis of MSc in medical-surgical nursing with the code of 3115 at Shahrekord University of Medical Sciences, Shahrekord, Iran.

\section{Conflict of interest}

The authors declare that they have no conflict of interest with respect to the research, authorship, and/or publication of this article.
The tele-nursing education by consultation or notification in Iran has used by phone in most of the studies (3). The health calling system through which the patients receive weekly or monthly calls from nurses is considered as a useful method of treatment in chronic diseases in an effective treatment outcome, better health status, development of patients-nurse communication, and overcome the wasting time and distance problems to provide better nursing cares (35). 


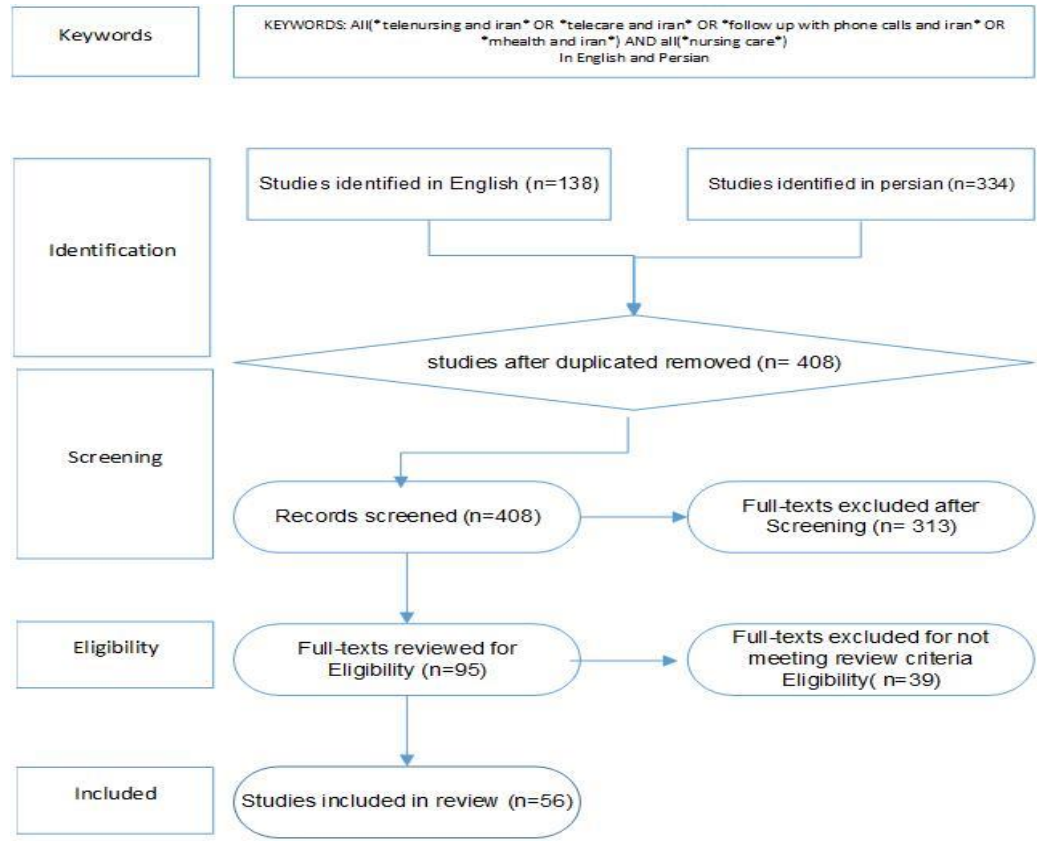

Figure 1. Flowchart of review

Table 2. Characteristics of the selected studies

\begin{tabular}{|l|l|l|l|l|l|}
\hline study & $\begin{array}{l}\text { Sample } \\
\text { size }\end{array}$ & Study design & $\begin{array}{l}\text { type of tele- } \\
\text { nursing }\end{array}$ & goal & outcome \\
\hline $\begin{array}{l}\text { Rezaei } \\
(2020) \\
(3)\end{array}$ & 90 & $\begin{array}{l}\text { clinical trial } \\
\text { with a pretest } \\
\text { posttest } \\
\text { design }\end{array}$ & telephone & $\begin{array}{l}\text { quality of life } \\
\text { (QOL) }\end{array}$ & $\begin{array}{l}\text { Intervention } \\
\text { improved the } \\
\text { quality of life of } \\
\text { survivors of burn } \\
\text { injuries }\end{array}$ \\
\hline $\begin{array}{l}\text { Bikmoradi } \\
(2016)(34)\end{array}$ & 71 & $\begin{array}{l}\text { quasi- } \\
\text { experimental } \\
\text { study }\end{array}$ & telephone & $\begin{array}{l}\text { Adherence of } \\
\text { treatment plan }\end{array}$ & $\begin{array}{l}\text { Tele-nursing } \\
\text { improve patients } \\
\text { adherence to } \\
\text { treatment plan. }\end{array}$ \\
\hline $\begin{array}{l}\text { Dadgari } \\
(2017)(35)\end{array}$ & 70 & RCT & telephone & quality of life & $\begin{array}{l}\text { this type of follow- } \\
\text { up counseling } \\
\text { could improve the } \\
\text { quality of life }\end{array}$ \\
\hline $\begin{array}{l}\text { Negarandeh } \\
(2019)(36)\end{array}$ & 80 & RCT & Telephone & $\begin{array}{l}\text { Self-Care Behaviors } \\
\text { and } \\
\text { Readmission }\end{array}$ & $\begin{array}{l}\text { tele-monitoring } \\
\text { improved self-care } \\
\text { behaviors but did } \\
\text { not reduce } \\
\text { readmission rates }\end{array}$ \\
\hline
\end{tabular}




\begin{tabular}{|c|c|c|c|c|c|}
\hline $\begin{array}{l}\text { Nazar mozafari } \\
(2016)(37)\end{array}$ & 50 & RCT & telephone & $\begin{array}{l}\text { nutritional self- } \\
\text { efficacy and } \\
\text { physical activity }\end{array}$ & $\begin{array}{l}\text { Intervention } \\
\text { improved } \\
\text { nutritional self- } \\
\text { efficacy and } \\
\text { physical activity }\end{array}$ \\
\hline $\begin{array}{l}\text { Namjoo Nasab } \\
(2017)(38)\end{array}$ & 64 & $\begin{array}{l}\text { experimental } \\
\text { deign }\end{array}$ & Telephone & Self-management & $\begin{array}{l}\text { Intervention } \\
\text { improved glucose } \\
\text { management, } \\
\text { dietary control, and } \\
\text { physical activity } \\
\text { but not health care } \\
\text { use }\end{array}$ \\
\hline $\begin{array}{l}\text { Sadeghi } \\
\text { shermeh } \\
(2013)(39)\end{array}$ & 99 & RCT & $\begin{array}{l}\text { telephone } \\
\text { and short } \\
\text { message } \\
\text { service }\end{array}$ & quality of life & $\begin{array}{l}\text { no significant } \\
\text { difference between } \\
\text { follow-up care by } \\
\text { telephone and short } \\
\text { massage services }\end{array}$ \\
\hline $\begin{array}{l}\text { Ghodsbin } \\
(2018)(40)\end{array}$ & 60 & RCT & telephone & ultrasound findings & $\begin{array}{l}\text { Intervention } \\
\text { improved liver size } \\
\text { and liver histology }\end{array}$ \\
\hline $\begin{array}{l}\text { Sabzevari } \\
(2014)(41)\end{array}$ & 50 & $\begin{array}{l}\text { quasi- } \\
\text { experimental }\end{array}$ & telephone & quality of life & $\begin{array}{l}\text { Intervention } \\
\text { improve the quality } \\
\text { of life }\end{array}$ \\
\hline $\begin{array}{l}\text { Parizad } \\
(2013)(42)\end{array}$ & 66 & experimental & telephone & self-care activities & $\begin{array}{l}\text { Intervention } \\
\text { promoted } \\
\text { self-care activities }\end{array}$ \\
\hline $\begin{array}{l}\text { Yekefallah } \\
(2016)(43)\end{array}$ & 100 & $\begin{array}{l}\text { quasi- } \\
\text { experimental }\end{array}$ & telephone & quality of life & $\begin{array}{l}\text { Intervention } \\
\text { improve the quality } \\
\text { of life }\end{array}$ \\
\hline $\begin{array}{l}\text { Mousavifar } \\
(2011)(44)\end{array}$ & 77 & $\begin{array}{l}\text { quasi- } \\
\text { experimental }\end{array}$ & $\begin{array}{l}\text { telephone } \\
\text { and short } \\
\text { message } \\
\text { service }\end{array}$ & $\begin{array}{l}\text { Adherence to } \\
\text { Treatment }\end{array}$ & $\begin{array}{l}\text { Interventions } \\
\text { improved } \\
\text { adherence to } \\
\text { Treatment and A1C } \\
\text { hemoglobin level }\end{array}$ \\
\hline $\begin{array}{l}\text { Borhani } \\
(2013)(45)\end{array}$ & 60 & RCT & $\begin{array}{l}\text { Cellphone } \\
\text { software }\end{array}$ & $\begin{array}{l}\text { A1c } \\
\text { hemoglobin }\end{array}$ & $\begin{array}{l}\text { Intervention had } \\
\text { positive effect on } \\
\text { metabolic control } \\
\text { of patients }\end{array}$ \\
\hline $\begin{array}{l}\text { Salimi Ezzt } \\
(2018)(46)\end{array}$ & 86 & $\begin{array}{l}\text { quasi- } \\
\text { experimental }\end{array}$ & telephone & $\begin{array}{l}\text { Treatment } \\
\text { Adherence and } \\
\text { Hemodialysis } \\
\text { Adequacy }\end{array}$ & $\begin{array}{l}\text { Intervention } \\
\text { improved treatment } \\
\text { adherence and } \\
\text { dialysis } \\
\text { adequacy } \\
\end{array}$ \\
\hline $\begin{array}{l}\text { Hemmati } \\
\text { Maslakpak } \\
(2012)(47)\end{array}$ & 66 & $\begin{array}{l}\text { Experimental } \\
\text { with a pretest } \\
\text { posttest } \\
\text { design }\end{array}$ & $\begin{array}{l}\text { telephone } \\
\text { and short } \\
\text { message } \\
\text { service }\end{array}$ & Glycemic Control & $\begin{array}{l}\text { Interventions } \\
\text { improved Glycemic } \\
\text { Control of patients }\end{array}$ \\
\hline $\begin{array}{l}\text { Fakharzadeh } \\
(2013)(48)\end{array}$ & 64 & $\mathrm{RCT}$ & telephone & $\begin{array}{l}\text { hemoglobin } \\
\text { (HbA1c) and } \\
\text { anthropometric } \\
\text { indexes }\end{array}$ & $\begin{array}{l}\text { tele-nursing, as a } \\
\text { follow-up, could } \\
\text { improve glycemic }\end{array}$ \\
\hline
\end{tabular}




\begin{tabular}{|c|c|c|c|c|c|}
\hline $\begin{array}{l}\text { Faraji } \\
(2015)(49)\end{array}$ & 70 & $\begin{array}{l}\text { non } \\
\text { randomized } \\
\text { controlled trial }\end{array}$ & telephone & $\begin{array}{l}\text { illness perception } \\
\text { and lifestyle }\end{array}$ & $\begin{array}{l}\text { Intervention } \\
\text { improved illness } \\
\text { perception but } \\
\text { lifestyle changes } \\
\text { requires long-term } \\
\text { interventions }\end{array}$ \\
\hline $\begin{array}{l}\text { Shojaee } \\
(2013)(50)\end{array}$ & 189 & $\begin{array}{l}\text { quasi- } \\
\text { experimental }\end{array}$ & telephone & level of hope & $\begin{array}{l}\text { Intervention } \\
\text { increased hope }\end{array}$ \\
\hline $\begin{array}{l}\text { Shojaee } \\
(2014)(30)\end{array}$ & 189 & $\mathrm{RCT}$ & telephone & anxiety & $\begin{array}{l}\text { Intervention } \\
\text { reduced anxiety }\end{array}$ \\
\hline $\begin{array}{l}\text { Zolfaghari } \\
(2017)(51)\end{array}$ & 32 & $\begin{array}{l}\text { quasi- } \\
\text { experimental }\end{array}$ & telephone & $\begin{array}{l}\text { Rehospitalization } \\
\text { and Depression }\end{array}$ & $\begin{array}{l}\text { Intervention } \\
\text { reduced short-term } \\
\text { depression and } \\
\text { decreases the } \\
\text { rehospitalization }\end{array}$ \\
\hline $\begin{array}{l}\text { Fallahpour } \\
(2018)(52)\end{array}$ & 60 & $\begin{array}{l}\text { quasi- } \\
\text { experimental }\end{array}$ & telephone & Fatigue & $\begin{array}{l}\text { Intervention } \\
\text { reduced the amount } \\
\text { and severity of } \\
\text { fatigue }\end{array}$ \\
\hline $\begin{array}{l}\text { Imani } \\
(2015)(53)\end{array}$ & 35 & $\begin{array}{l}\text { before-after } \\
\text { semi-empirical }\end{array}$ & telephone & anxiety level & $\begin{array}{l}\text { Intervention } \\
\text { reduced the level of } \\
\text { anxiety }\end{array}$ \\
\hline $\begin{array}{l}\text { Najafi } \\
(2017)(29)\end{array}$ & 100 & RCT & $\begin{array}{l}\text { Telephone } \\
\text { and telegram }\end{array}$ & blood pressure & $\begin{array}{l}\text { Interventions were } \\
\text { effective on the } \\
\text { blood pressure }\end{array}$ \\
\hline $\begin{array}{l}\text { Forouzesh } \\
(2017)(54)\end{array}$ & 128 & RCT & telephone & $\begin{array}{l}\text { readmission due to } \\
\text { complications }\end{array}$ & $\begin{array}{l}\text { Intervention was } \\
\text { effective on the } \\
\text { number of visits } \\
\text { due to } \\
\text { postoperative } \\
\text { complications }\end{array}$ \\
\hline $\begin{array}{l}\text { Bikmoradi } \\
(2020)(55)\end{array}$ & 70 & RCT & telephone & Pain Reduction & $\begin{array}{l}\text { Intervention } \\
\text { reduced pain and } \\
\text { improved patient } \\
\text { health }\end{array}$ \\
\hline $\begin{array}{l}\text { Behzad } \\
(2016)(56)\end{array}$ & 88 & $\begin{array}{l}\text { quasi- } \\
\text { experimental }\end{array}$ & telephone & $\begin{array}{l}\text { Self - Efficacy in } \\
\text { self-care behaviors }\end{array}$ & $\begin{array}{l}\text { Intervention } \\
\text { promoted the Self - } \\
\text { Efficacy in self- } \\
\text { care behaviors }\end{array}$ \\
\hline $\begin{array}{l}\text { Kamrani } \\
(2015)(57)\end{array}$ & 90 & RCT & telephone & $\begin{array}{l}\text { adherence to } \\
\text { treatment }\end{array}$ & $\begin{array}{l}\text { Intervention } \\
\text { improved } \\
\text { adherence to } \\
\text { treatment }\end{array}$ \\
\hline $\begin{array}{l}\text { Ebrahimi } \\
(2017)(58)\end{array}$ & 60 & RCT & telephone & medical adherence & $\begin{array}{l}\text { Intervention } \\
\text { increased medical } \\
\text { adherence }\end{array}$ \\
\hline $\begin{array}{l}\text { Estaji } \\
(2016)(59)\end{array}$ & 30 & $\begin{array}{l}\text { before and after } \\
\text { quasi- } \\
\text { experimental }\end{array}$ & $\begin{array}{l}\text { short } \\
\text { message } \\
\text { service }\end{array}$ & compliance & $\begin{array}{l}\text { Intervention was } \\
\text { effective on } \\
\text { compliance of } \\
\text { patients }\end{array}$ \\
\hline $\begin{array}{l}\text { Zamanzadeha } \\
(2017)(60)\end{array}$ & 66 & $\begin{array}{l}\text { single-blind } \\
\text { RCT }\end{array}$ & $\begin{array}{l}\text { telephone } \\
\text { and short } \\
\text { message } \\
\text { service }\end{array}$ & empowerment & $\begin{array}{l}\text { Interventions } \\
\text { significantly } \\
\text { improved the } \\
\text { empowerment }\end{array}$ \\
\hline
\end{tabular}




\begin{tabular}{|c|c|c|c|c|c|}
\hline $\begin{array}{l}\text { Kargar Jahromi } \\
(2016)(31)\end{array}$ & 60 & $\begin{array}{l}\text { double blind } \\
\text { RCT }\end{array}$ & telephone & $\begin{array}{l}\text { Depression, Anxiety } \\
\text { and Stress }\end{array}$ & $\begin{array}{l}\text { Intervention } \\
\text { improved } \\
\text { emotional and } \\
\text { health status of } \\
\text { patients }\end{array}$ \\
\hline $\begin{array}{l}\text { Pedram Razi } \\
(2013)(61)\end{array}$ & 98 & $\begin{array}{l}\text { quasi- } \\
\text { experimental } \\
\text { controlled } \\
\text { clinical trial }\end{array}$ & $\begin{array}{l}\text { short } \\
\text { message } \\
\text { service }\end{array}$ & $\begin{array}{l}\text { Asthma Self- } \\
\text { Management }\end{array}$ & $\begin{array}{l}\text { Intervention } \\
\text { improved Asthma } \\
\text { Self-Management }\end{array}$ \\
\hline $\begin{array}{l}\text { Najafi } \\
(2016)(62)\end{array}$ & 100 & $\begin{array}{l}\text { non-blinded } \\
\text { randomized } \\
\text { controlled } \\
\text { clinical trial }\end{array}$ & telephone & $\begin{array}{l}\text { Medication and } \\
\text { Dietary Adherence }\end{array}$ & $\begin{array}{l}\text { Intervention had } \\
\text { positive effects on } \\
\text { adherence to } \\
\text { dietary and } \\
\text { medication regimen } \\
\text { in the patients with } \\
\text { MI }\end{array}$ \\
\hline $\begin{array}{l}\text { Poorgholami } \\
(2016)(63)\end{array}$ & 75 & $\begin{array}{l}\text { single-blind } \\
\text { RCT }\end{array}$ & telephone & Level of Hope & $\begin{array}{l}\text { teaching followed } \\
\text { by telephone } \\
\text { follow-up was } \\
\text { associated with } \\
\text { higher levels of } \\
\text { hope. }\end{array}$ \\
\hline $\begin{array}{l}\text { Malakouti } \\
(2016)(64)\end{array}$ & 182 & $\begin{array}{l}\text { Multicenter } \\
\text { RCT }\end{array}$ & telephone & $\begin{array}{l}\text { Clinical } \\
\text { Outcomes }\end{array}$ & $\begin{array}{l}\text { telephone follow- } \\
\text { up services } \\
\text { improved Most } \\
\text { clinical variables }\end{array}$ \\
\hline $\begin{array}{l}\text { Bikmoradi } \\
(2017)(65)\end{array}$ & 71 & $\begin{array}{l}\text { quasi- } \\
\text { experimental }\end{array}$ & telephone & quality of life & $\begin{array}{l}\text { Intervention } \\
\text { improved the } \\
\text { quality of life }\end{array}$ \\
\hline $\begin{array}{l}\text { Samimi } \\
(2018)(66)\end{array}$ & 70 & $\begin{array}{l}\text { quasi- } \\
\text { experimental }\end{array}$ & telephone & Self-care & $\begin{array}{l}\text { Intervention } \\
\text { improved total } \\
\text { self-care and } \\
\text { glycosylated } \\
\text { hemoglobin }\end{array}$ \\
\hline $\begin{array}{l}\text { Boroumand } \\
(2016)(67)\end{array}$ & 70 & RCT & $\begin{array}{l}\text { telephone } \\
\text { and short } \\
\text { message } \\
\text { service }\end{array}$ & cardiac self-efficacy & $\begin{array}{l}\text { Interventions } \\
\text { promoted the } \\
\text { cardiac } \\
\text { self-efficacy of } \\
\text { patients }\end{array}$ \\
\hline $\begin{array}{l}\text { Khodaparast } \\
(2018)(15)\end{array}$ & 60 & $\mathrm{RCT}$ & $\begin{array}{l}\text { Telegram } \\
\text { and } \\
\text { multimedia } \\
\text { CD }\end{array}$ & $\begin{array}{l}\text { the } \\
\text { Success of Lactation }\end{array}$ & $\begin{array}{l}\text { Interventions had } \\
\text { similar effects } \\
\text { on the behavior of } \\
\text { mother and baby in } \\
\text { breastfeeding, } \\
\text { effective } \\
\text { nutrition of infant, } \\
\text { frequency and the } \\
\text { period of } \\
\text { breastfeeding. }\end{array}$ \\
\hline $\begin{array}{l}\text { Jalal Marvi } \\
(2019)(28)\end{array}$ & 91 & RCT & $\begin{array}{l}\text { Education } \\
\text { DVD and } \\
\text { telephone }\end{array}$ & Severity of Insomnia & $\begin{array}{l}\text { Telehealth reduced } \\
\text { pregnant women } \\
\text { insomnia }\end{array}$ \\
\hline
\end{tabular}


Tele-Nursing strategies in Iran

\begin{tabular}{|c|c|c|c|c|c|}
\hline $\begin{array}{l}\text { Zolfaghari } \\
(2012)(27)\end{array}$ & 77 & $\begin{array}{l}\text { quasi- } \\
\text { experimental }\end{array}$ & $\begin{array}{l}\text { short } \\
\text { message } \\
\text { services and } \\
\text { telephone }\end{array}$ & diabetic adherence & $\begin{array}{l}\text { Interventions } \\
\text { improved HbA1c } \\
\text { levels and } \\
\text { adherence to } \\
\text { diabetes therapeutic } \\
\text { regimen for three } \\
\text { months }\end{array}$ \\
\hline $\begin{array}{l}\text { Zarei } \\
(2013)(26)\end{array}$ & 60 & RCT & telephone & Asthma Control & $\begin{array}{l}\text { Educational } \\
\text { Intervention and } \\
\text { Modifying } \\
\text { (telephone follow } \\
\text { up) } \\
\text { improved the } \\
\text { asthma control }\end{array}$ \\
\hline $\begin{array}{l}\text { Niksalehi } \\
(2018)(68)\end{array}$ & 54 & $\begin{array}{l}\text { pre-test and } \\
\text { post-test } \\
\text { clinical study }\end{array}$ & $\begin{array}{l}\text { short } \\
\text { message } \\
\text { services }\end{array}$ & $\begin{array}{l}\text { Supporting } \\
\text { Intervention }\end{array}$ & $\begin{array}{l}\text { Intervention } \\
\text { improved mothers' } \\
\text { health literacy } \\
\text { about maternal } \\
\text { postpartum } \\
\text { psychological } \\
\text { disorders }\end{array}$ \\
\hline $\begin{array}{l}\text { Ahmadi babadi } \\
(2017)(69)\end{array}$ & 60 & $\begin{array}{l}\text { quasi- } \\
\text { experimental }\end{array}$ & telephone & $\begin{array}{l}\text { the feeling of } \\
\text { loneliness }\end{array}$ & $\begin{array}{l}\text { Telephone follow- } \\
\text { up decreased the } \\
\text { feeling of } \\
\text { loneliness }\end{array}$ \\
\hline $\begin{array}{l}\text { Roozbahani } \\
(2015)(70)\end{array}$ & 80 & RCT & telephone & $\begin{array}{l}\text { blood glucose levels } \\
\text { and postpartum } \\
\text { screening }\end{array}$ & $\begin{array}{l}\text { Intervention } \\
\text { reduced fasting } \\
\text { blood glucose } \\
\text { levels and also } \\
\text { increased the rate } \\
\text { of } \\
\text { postpartum } \\
\text { screening test }\end{array}$ \\
\hline $\begin{array}{l}\text { Yekefallah } \\
(2016)(71)\end{array}$ & 100 & $\begin{array}{l}\text { quasi- } \\
\text { experimental }\end{array}$ & telephone & quality of life & $\begin{array}{l}\text { intervention } \\
\text { increased the } \\
\text { patients' quality of } \\
\text { life and could } \\
\text { provide better } \\
\text { nursing care }\end{array}$ \\
\hline $\begin{array}{l}\text { Najafi } \\
\text { Ghezeljeh } \\
\text { (2018) (72) }\end{array}$ & 100 & RCT & $\begin{array}{l}\text { telephone } \\
\text { and telegram }\end{array}$ & $\begin{array}{l}\text { Self-Management } \\
\text { Behaviors }\end{array}$ & $\begin{array}{l}\text { Interventions } \\
\text { improved Self- } \\
\text { Management } \\
\text { Behaviors }\end{array}$ \\
\hline $\begin{array}{l}\text { Nesari } \\
(2010)(73)\end{array}$ & 61 & Not mentioned & telephone & $\begin{array}{l}\text { adherence to a } \\
\text { diabetes } \\
\text { therapeutic regimen }\end{array}$ & $\begin{array}{l}\text { Intervention was } \\
\text { effective in } \\
\text { enhancing the level } \\
\text { of adherence to a } \\
\text { diabetes } \\
\text { therapeutic } \\
\text { regimen, such that } \\
\text { the HbAlc level } \\
\text { decreased }\end{array}$ \\
\hline
\end{tabular}


Fathizadeh Dehkordi, P. et al

\begin{tabular}{|c|c|c|c|c|c|}
\hline $\begin{array}{l}\text { Mohammadi } \\
(2017)(8)\end{array}$ & 109 & $\begin{array}{l}\text { quasi- } \\
\text { experimental }\end{array}$ & telephone & self-efficacy & $\begin{array}{l}\text { self-efficacy was } \\
\text { significantly } \\
\text { increased after the } \\
\text { intervention }\end{array}$ \\
\hline $\begin{array}{l}\text { Hemmati } \\
\text { Maslakpak } \\
\text { (2014)(74) }\end{array}$ & 80 & $\begin{array}{l}\text { quasi- } \\
\text { experimental }\end{array}$ & telephone & Self-Efficacy & $\begin{array}{l}\text { Intervention } \\
\text { increased the } \\
\text { perception of } \\
\text { self-efficacy }\end{array}$ \\
\hline $\begin{array}{l}\text { Aliabadi } \\
(2015)(75)\end{array}$ & 60 & RCT & telephone & $\begin{array}{l}\text { knowledge, attitude } \\
\text { and skills of } \\
\text { caregiving }\end{array}$ & $\begin{array}{l}\text { Intervention } \\
\text { increased } \\
\text { awareness, } \\
\text { understanding and } \\
\text { skills of brain } \\
\text { damaged } \\
\text { caregivers }\end{array}$ \\
\hline $\begin{array}{l}\text { Parizad } \\
(2014)(76)\end{array}$ & 66 & $\begin{array}{l}\text { experimental } \\
\text { study }\end{array}$ & $\begin{array}{l}\text { telephone } \\
\text { and short } \\
\text { message } \\
\text { services }\end{array}$ & $\begin{array}{l}\text { Laboratory } \\
\text { Parameters }\end{array}$ & $\begin{array}{l}\text { Intervention had } \\
\text { effect on } \\
\text { controlling } \\
\text { the laboratory } \\
\text { parameters }\end{array}$ \\
\hline $\begin{array}{l}\text { Safari } \\
(2017)(33)\end{array}$ & 64 & $\begin{array}{l}\text { Double-blind } \\
\text { RCT }\end{array}$ & telephone & $\begin{array}{l}\text { the amount of } \\
\text { glycosylated } \\
\text { hemoglobin }\end{array}$ & $\begin{array}{l}\text { Intervention had } \\
\text { effects on patients' } \\
\text { hemoglobin A1C } \\
\text { and fasting blood } \\
\text { sugar control }\end{array}$ \\
\hline $\begin{array}{l}\text { Madadkar } \\
\text { Dehkordi } \\
(2020)(4)\end{array}$ & 60 & RCT & telephone & Quality of Life & $\begin{array}{l}\text { Intervention } \\
\text { significantly } \\
\text { increased the } \\
\text { quality of Life }\end{array}$ \\
\hline $\begin{array}{l}\text { Poshtchaman } \\
(2014)(77)\end{array}$ & 90 & RCT & $\begin{array}{l}\text { telephone } \\
\text { and short } \\
\text { message } \\
\text { services }\end{array}$ & treatment adherence & $\begin{array}{l}\text { Interventions } \\
\text { improved treatment } \\
\text { adherence }\end{array}$ \\
\hline $\begin{array}{l}\text { Zolfaghari } \\
(2012)(13)\end{array}$ & 77 & $\begin{array}{l}\text { Semi } \\
\text { experimental } \\
\text { study }\end{array}$ & $\begin{array}{l}\text { telephone } \\
\text { and short } \\
\text { message } \\
\text { services }\end{array}$ & HbA1c & $\begin{array}{l}\text { Interventions } \\
\text { improved } \mathrm{HbA} 1 \mathrm{c}\end{array}$ \\
\hline
\end{tabular}


Table 3. Method of investigations

\begin{tabular}{|c|c|}
\hline Intervention & Percent of intervention \\
\hline Follow-up after an educational or empowerment program & $66 \%$ \\
\hline Follow-up with education or consultation & $12.5 \%$ \\
\hline Education, consultation or notification & $21.5 \%$ \\
\hline
\end{tabular}

Table 4. Results of review

\begin{tabular}{|c|c|}
\hline Method of tele-nursing & Percent of usage \\
\hline Telephone & $74 \%$ \\
\hline SMS & $17 \%$ \\
\hline Software & $2 \%$ \\
\hline Telegram & $4 \%$ \\
\hline CD-DVD & $3 \%$ \\
\hline
\end{tabular}

\section{References}

1. Chegini, Z., Jafari-Koshki, T., Kheiri, M., Behforoz, A., Aliari, S., Mitra, U., Shariful Islam, S.M. Missed Nursing Care and Related Factors in Iranian Hospitals : A Cross Sectional Survey. J Nurs Manag 2020; Published date: 2020-05-30. doi:10.1111/jonm.13055.

2. Ghoulami-shilsari, F., Esmaeilpour Bandboni, M. Tele-Nursing in Chronic Disease Care : A Systematic Review. Jundishapur J Chronic Dis Care 2019; 8(2):e84379.

3. Rezaei, M., Jalali, R., Heydarikhayat, N. Effect of Tele-nursing and Face-to-Face Training Techniques on Quality of Life in Burn Patients: A Clinical Trial. Arch Phys Med Rehabil 2020; 101(4):667-73.

4. Madadkar Dehkordi, S., Noorian, K. Effect of Multimedia Education Based on the Familycentered Ap- proach and Telephone Follow-up ( Telenursing ) on the Quality of Life of Patients with Myocardial Infarction after Discharge. J Clin Nurs Midwifery 2020; 4(3):500-510.

5. Purabdollah, M., Ghasempour, M. Telenursing new opportunity for nursing care in covid-19 pandemic crisis. Iran J Public Health 2020; 49:130-1.

6. Ramadan, R., Hussein, E., Amr, T. Telenursing of Osteoporosis Self-Management for Women Health. Egypt J Nurs Health Sci 2020; 1(1):99-118.
7. de Lima Silva, K., Évora, Y.D.M., Cintra C.S.J. Software development to support decision making in the selection of nursing diagnoses and interventions for children and adolescents. Rev Latino-Am Enferm 2015; 23(5):927-35.

8. Mohammadi, N., Omidi, A., Soleymani, R., Roshanaei, G. The Effect of Telephone Nursing Follow-up on Self-Ef- ficacy of Females With Type 2 Diabetes Mellitus. Avicenna J Nurs Midwifery Care 2017; 25(2):61-68.

9. Schlottmann, H., Broome, M., Herbst, R., Burkhardt, M.C., Mescher, A. Nurse-Led Telephone Follow-Up to Improve Parent Promotion of Healthy Behaviors in Young Children With Motivational Interviewing Techniques. J Pediatr Health Care 2019; 33(5):1-10.

10. Mohamed, S.A., Fashafsheh, I.H. Effect of Educational Intervention and Telephone Follow-Up Program on Knowledge, Practice and Quality Of Life among Patients with Urinary Diversion: A QuasiExperimental Study. Int J Nurs 2019; 6(1):58-71.

11. Sandelius, S., Wahlberg, A.C. Telenurses' experiences of monitoring calls to parents of children with gastroenteritis. Scand J Caring Sci 2020; 34(3):658-665.

12. Moradi, A., Mohammad, S., Salimi, M., Nouhjah, S. The effect of short message service (SMS) on knowledge and preventive behaviors of diabetic foot ulcer in patients with diabetes type 2. Diabetes Metab Syndr 2019; 13(2):1255-60. 
13. Zolfaghari, M., Mousavifar, S.A, Haghani, H. Mobile phone text messaging and Telephone follow-up in type 2 diabetic patients for 3 months: a comparative study. J Diabetes Metab Disord 2012; 11(1):7.

14. Schwebel, F.J., Larimer, M.E. Using text message reminders in health care services : A narrative literature review. Internet Interv 2018; 13:82-104.

15. Khodaparast, M., Rahani, T., Sadeghi, T., Boskabadi, H., Yavari, M. The Effect of Training Through Tele-nursing and Multimedia on the Success of Lactation in Preterm Infants After Discharge. J North Khorasan University Med Sci 2018; 10(2):1320.

16. Alvarez, A.G, Dal Sasso, G.T.M., Iyengar, M.S. Persuasive technology in teaching acute pain assessment in nursing: Results in learning based on pre and post-testing. Nurse Educ Today 2017; 50:109114.

17. Wang, T., Huang, R., Yang, S., Chou, C. Evaluating the Effects of a Mobile Health App on Reducing Patient Care Needs and Improving Quality of Life After Oral Cancer Surgery : Quasiexperimental Study. JMIR Mhealth Uhealth. 2020; 8(7):e18132.

18. Mazlom, S.R., Rajabpoor, M. Development and Assessment of Computerized Software for Nursing Process: a Step toward Promotion of Nursing Education and Care. Iran J Med Educ 2014; 14(4):312322.

19. Finkelstein, S.M., Celebrezze, M., Cady, R., Lunos, S., Looman, W.S. Strategies to Maximize Data Collection Response Rates in a Randomized Control Trial Focused on Children with Medical Complexity. Telemed J E Health 2016; 22(4):295-301.

20. Hawkins, J.E. The Practical Utility and Suitability of Email Interviews in Qualitative Research. The Qualitative Report 2018; 23(2):493501.

21. Kawaguchi, T., Azuma, M., Ohta, K. Development of a tele-nursing system for patients with chronic conditions. J Telemed Telecare 2004; 10(4):239-44.

22. de Souza-junior, V.D., Costa Mendes, I.A., Mazzo, A., de Godoy, S., Dos Santos, A.C. Telenursing Intervention for Clean Intermittent Urinary. Comput Inform Nurs 2017; 35(12):653-660.

23. Javadi Yeganeh, M., Kosari, M., Kheyrkhah, T. Cell Phone and Its Functions for Iranian Users; Focusing on Gender Differences. Q Soc Stud Res Iran.
$2012 ; 1(2): 23-54$.

24. Some $64 \%$ of Iranian are internet users:report. Tehran Times 2019; Janurary 11. Available from: https://www.tehrantimes.com/news/431713/Some64-of-Iranians-are-internet-users-report

25. Ershad Sarabi, R., Sadoughi, F., Orak Rouhangiz, J., Bahaadinbeigy, K. Role of Mobile Technology in Iran Healthcare System: A review study. J Health Biomed Info 2018; 4(4):313-326.

26. Zarei, S., Valizadeh, L., Bilan, N. The Effect of Educational and Modifying Intervention on Asthma Control among Adolescents : a Randomized Clinical Trial. J Caring Sci 2013; 2(1):73-82.

27. Zolfaghari, M., Mousavifar, S.A., Pedram, S., Haghani, H. The impact of nurse short message services and telephone follow-ups on diabetic adherence:which one is more effective? J Clin Nurs 2012; 21:1922-31.

28. Jalal Marvi, F., Kordi, M., Mazlom, S.R., Rezaei Talab, F. Comparing the Effect of Training Based on Continuous Care Model and Telehealth on Severity of Insomnia in Pregnant Women. J North Khorasan Med Sci 2019; 11(3):38-45.

29. Najafi Ghezeljeh, T., Nasr Esfahani, M., Sharifian, S. Comparing the effect of self-management training by mobile phone- based social network follow-up on blood pressure in people with hypertension. Cardiovasc Nurs J 2017; 6(1):33-42.

30. Shojaee, A., Nehrir, B., Naderi, N., Zareyan, A. Comparison of patient education and follow up by nurse on anxiety in heart failure patients. Med Surg Nurs J 2014; 3(1):52-60.

31. Kargar Jahromi, M., Javadpour, S., Taheri, L., Poorgholami, F. Effect of Nurse-Led Telephone Follow-ups (Tele-Nursing) on Depression, Anxiety and Stress in Hemodialysis Patients. Glob J Health Sci 2016; 8(3):168-73.

32. Ajalli, A., Fallahi Khoshknab, M. Tele nursing care in chronic patients: a systematic review. Iran J Rehabil Res Nurs 2015; 1(3):76-86.

33. Safari, A., Sanagoo, A., Kavosi, A., Behnampoor, N., Jouybari, L. The comparative effect of insulin training with and without phone calls follow-up on the amount of glycosylated hemoglobin in people with type 2 diabetes. J Urmia Nurs Midwifery Fac 2017; 15(7):504-513.

34. Bikmoradi, A., Masmouei, B., Ghomeisi, M., 
Roshanaei, G. Impact of Tele-nursing on adherence to treatment plan in discharged patients after coronary artery bypass graft surgery: A quasi-experimental study in Iran. Int J Med Inform 2016; 86:43-8.

35. Dadgari, F., Hoseini, S., Aliyari, S., Masoudi, S. The effect of sustained nursing consulting via telephone (Tele Nursing) on the quality of life in hypertensive patients. Appl Nurs Res 2017; 35:10611.

36. Negarandeh, R. Evaluating the Effect of Monitoring through Telephone (Tele-Monitoring) on Self-Care Behaviors and Readmission of Patients with Heart Failure after Discharge. Appl Clin Inform 2019; 10:261-268.

37. Nazar Mozafari, M., Jahani, Y., Saied Najafi, S., Hosein Rezaei, H. Effect of telephone follow-up (tele-nursing) on nutritional self-efficacy and physical activity in patients with coronary artery bypass graft in shiraz namazy hospital in 2015. Iran J Anesthesiol Crit Care 2016; 2(4):53-63.

38. Nasab, M.N., Ghavam, A., Yazdanpanah, A., Jahangir, F., Shokrpour, N. Effects of Selfmanagement Education Through Telephone Followup in Diabetic Patients. Health Care Manag 2017; 36(3):273-81.

39. Sadeghi, M., Ghafouri, F., Tadrisi, S.D., Tayyebi, A. The effect of follow-up care by telephone and short massage services on patient's quality of life after cardiac valve replacement surgery. Iran J Crit Care Nurs 2013; 6(1):65-72.

40. Ghodsbin, F., Javanmardifard, S., Kaviani, M.J., Jahanbin, I. Effect of tele-nursing in the the improving of the ultrasound findings in patients with nonalcoholic fatty liver diseases: A Randomized Clinical Trial study: Effect of tele-nursing. Invest Educ Enferm 2018; 36(3):e09.

41. Sabzevari, S., Lashkari, T., Borhani, F., Abbaszadeh, A. Effect of phone follow-up on quality of life in type II diabetic patients. Med Surg Nurs J 2014; 3(2):84-90.

42. Parizad, N., Hemmati Maslakpak, M., Khalkhali, H. Promoting Self-Care in Patients with Type 2 Diabetes: Tele-Education. Hakim Res J 2013; 16(3):220-227.

43. Yekefallah, L., Najaf, M., Shahrokhi, A., Javadi, H., Mohammadpoorasl, A. Effect of telenursing on quality of life of patients with atrial fibrillation referred to the teaching hospitals in Qazvin. J Qazvin Univ Med Sci 2016; 20(1):56-62.
44. Mousavifar, A., Zolfaghari, M., Pedram, S., Haghani, H. The Effectiveness of two follow-up methods (mobile and telephone) on Adherence to Treatment indiabetics. Iran J Diabetes Lipid Disord 2011; 10(4):407-418.

45. Borhani, F., Ranjbar, H., Abbaszadeh, A., Abazari, F., Ranjbar, A. The Effect of Tele-nursing (Cellphone software) on A1c hemoglobin in patients with Type 2 Diabetes Mellitus. J Army Univ Med Sci. 2013; 11(2):130-137.

46. Salimi Ezzat, L., Hanifi, N., Dinmohammadi, M. Effect of Telephone Consultation and Follow-up on Treatment Adherence and Hemodialysis Adequacy in Hemodialysis Patients. J Maz Univ Med Sci. 2018; 27(157):157-170.

47. Hemmati Maslakpak, M., Parizad, N., Khalkhali, H. The Effect of Tele-Education By Telephone And Short Message Service On Glycaemic Control In Patient With Type 2 Diabetes. Nurs Midwifery J. 2012; 10(4):580-588.

48. Fakharzadeh, L., Shahbazian, H., Salehinia, H., Yaghoobi, M., Haghighizade, M.H., Karandish, M. Effect of tele-nursing on glycosylated hemoglobin (HbA1c) and anthropometric indexes in type 2 diabetic patients. Mod Care, Sci Q Birjand Nurs Midwifery Fac. 2013; 10(2):101-107.

49. Faraji, N., Pashaeypoor, S., Negarandeh, R. The effect of pre-discharge education and telephone follow- up on illness perception and lifestyle in patients with myocardial infarction. Sci J Hamadan Nurs Midwifery Fac. 2015; 23(3):82-91.

50. Shojaee, A., Nehrir, B., Naderi, N., Zareiyan, A. Effect of patient education and telephone follow up by the nurse on the level of hope in patients suffering from heart failure. Nurs Educ. 2013; 2(3):16-26.

51. Zolfaghari, A., Dadgari, F., Farsi, Z. Effect of Telephone Follow-up Care on Rehospitalization and Depression in Patients Undergoing Open Heart Surgery in a Selected Military Hospital. MCS. 2017; 4(2):129-137.

52. Fallahpour, S., Nasiri, M., Fotokian, Z., Alipour, Z.J., Haji Ahmadi, M. The Effect of Telephone Follow up ( Tele-nursing ) on Fatigue in the Elderly Undergoing Hemodialysis. J Crit Care Nurs. 2018; 11(4):1-10.

53. Imani, A., Dabirian, A., Safavi Bayat, Z., Payandeh, A. Examining the impact of nurse notification by phone (tele-nursing) on anxiety level of hospitalized patient's family in intensive care unit. 
Iran J Nurs Res. 2015; 9(4):22-28.

54. Forouzesh, M., Sanagoo, A., Ghavidel, A., Vakili, M.A., Jouybari, L., Araghian Mojarad, F. The effect of tele-nursing (telephone foolow up) after discharge on readmission due to complications after coronary artery bypass graft surgery. J Urmia Nurs Midwifery Fac. 2017; 15(5):584-594.

55. Bikmoradi, A., Abdolmalaki, S., Tapak, L. The Effect of Telephone Consultation and Follow-up on Pain Reduction in Multiple Sclerotic Patients : a Randomized Clinical Trial. Avicenna J Nurs Midwifery Care. 2020; 28(1):11-19.

56. Behzad, Y., Bastani, F., Haghani, H. Effect of empowerment program with the telephone followup (tele-nursing) on self-efficacy in self-care behaviors in hypertensive older adults. J Urmia Nurs Midwifery Fac. 2016; 3(11):1004-1015.

57. Kamrani, F., Nikkhah, S., Borhani, F., Jalali, M., Shahsavari, S., Nirumand-Zandi, K. The effect of patient education and nurse-led telephone follow-up (tele-nursing) on adherence to treatment in patients with acute coronary syndrome. Cardiovasc Nurs Journa. 2015; 4(3):16-24.

58. Ebrahimi, S., Zakerimoghadam, M., Shahsavari, H., Gholizadeh, B., Naboureh, A. Effects of self-management program and telephone follow up on medical adherence in patients with ischemic heart disease. Koomesh. 2017; 19(1):213-219.

59. Estaji, Z., Hejazi, S., Tabarraie, Y., Saedi, M. The Effects of training through text messaging via cell phones on the compliance of patients undergoing hemodialysis. J North Khorasan Univ Med Sci. 2016; 8(2):203-213.

60. Zamanzadeh, V., Zirak, M., Hemmati, M. Diabetes \& Metabolic Syndrome: Clinical Research \& Reviews Distance education and diabetes empowerment: A single-blind randomized control trial. Diabetes Metab Syndr Clin Res Rev. 2017; 11:S247-51.

61. Pedram Razi, S., Piroozmand, N., Zolfaghari, M., Kazemnejad, A., Firoozbakhsh, S. Education of How-to-Use Peak Flow Meter and Following up via SMS on Asthma Self-Management. Hayat. 2013; 18(4):19-27.

62. Najafi, S.S., Shaabani, M., Momennassab, M., Aghasadeghi, K. The Nurse-Led Telephone Follow-Up on Medication and Dietary Adherence among Patients after Myocardial Infarction: A Randomized Controlled Clinical Trial. Int J
Community Based Nurs Midwifery. 2016; 4(3):199208.

63. Poorgholami, F., Mansoori, P., Montaseri, Z., Najafi, K. Effect of Self Care Education with and without Telephone Follow-Up on the Level of Hope in Renal Dialysis Patients : A Single-Blind Randomized Controlled Clinical Trial. Int J Community Based Nurs Midwifery. 2016; 4(3):256-64.

64. Malakouti, S.K., Nojomi, M., Mirabzadeh, A., Mottaghipour, Y., Zahiroddin, A., Mohammadi Kangran, H. A Comparative Study of Nurses as Case Manager and Telephone Follow-up on Clinical Outcomes of Patients with Severe Mental Illness. Iran J Med Sci January. 2016; 41(1):19-27.

65. Bikmoradi, A., Masmouei, B., Ghomeisi, M., Roshanaei, G., Masiello, I. Patient Education and Counseling Impact of telephone counseling on the quality of life of patients discharged after coronary artery bypass grafts. Patient Educ Couns. 2017; 100(12):2290-6.

66. Samimi, Z., Talakoub, S., Ghazavi, Z. Effect of Telephone Follow - up by Nurses on Self - care in Children with Diabetes. Iran J Nurs Midwifery Res. 2018; 23:26-30.

67. Boroumand, S., Moeini, M. The effect of a text message and telephone follow-up program on cardiac self-efficacy of patients with coronary artery disease: A randomized controlled trial. Iran J Nurs Midwifery Res. 2016; 21:171-6.

68. Niksalehi, S., Taghadosi, M., Mazhariazad, F., Tashk, M. The effectiveness of mobile phone text massaging support for mothers with postpartum depression : A clinical before and after study. J Fam Med Prim Care. 2018; 7:1058-62.

69. Ahmadi Babadi, S., Sadrgh Moghadam, L., Delshad Noghabi, A. Comparing the effectiveness of tele-nursing with in-person follow up on the feeling of loneliness among the elderly in community health centers in Ahvaz in 2017. J Gerontol. 2017; 2(2):5865.

70. Roozbahani, R.K., Geranmayeh, M., Hantoushzadeh, S., Mehran, A. Effects of telephone follow-up on blood glucose levels and post- partum screening in mothers with Gestational Diabetes Mellitus. Med J Islam Repub Iran. 2015; 29(249):1-6.

71. Yekefallah, L., Najafi, M., Shahrokhi, A., Javadi, H., Mohammadpoorasl, A. Effect of telenursing on quality of life of patients with atrial fibrillation referred to the teaching hospitals in 
Qazvin. J Qazvin Univ Med Sci. 2016; 20(1):56-62.

72. Najafi Ghezeljeh, T., Sharifian, S., Nasr Isfahani, M., Haghani, H. Comparing the Effects of Education Using Telephone Follow-Up and Smartphone-Based Social Networking Follow-Up on Self-Management Behaviors among Patients with Hypertension. Contemp Nurse. 2018; 54(4-5):362373.

73. Nesari, M., Zakerimoghadam, M., Rajab, A., Bassampour, S., Faghihzadeh, S. Effect of telephone follow-up on adherence to a diabetes therapeutic regimen. japan J Nurs Sci. 2010; 7:121-8.

74. Hemmati Maslakpak, M., Raiesi, Z. Effect of a Self-Management and Follow-Up Program on SelfEfficacy in Patients With Multiple Sclerosis: A Randomized Clinical Trial. Nurs Midwifery Stud. 2014; 3(4):e25661.

75. Aliabadi, M., Mojali, M., Khosravan, S., Mohammadzadeh, F. The effect of empowering family caregivers of brain damaged patients base on problem solving by telephone follow-ups on knowledge, attitude and skills of caregiving. Iran J Rehabil Res Nurs. 2015; 1(2):10-21.

76. Parizad, N., Hemmati Maslakpak, M., Khalkhali, H. The Effect of Tele-Education by Telephone and Short Message Service on Laboratory Parameters in Patients with Type 2 Diabetes. J Ardabil Univ Med Sci. 2014; 14(1):7-17.

77. Poshtchaman, Z., Jadid Milani, M., Atashzadeh Shoorideh, F., Akbarzadeh Bagheban, A. The effect of two ways of using the phone and SMS follow-up care on treatment adherence in Coronary Artery Bypass Graft patients. Cardiovasc Nurs J. 2014; 3(2):6-14. 